\title{
QSM based NOMA for Multi-User Wireless Communication
}

\author{
Rahmat Faddli Siregar, Nandana Rajatheva, and Matti Latva-aho \\ Centre for Wireless Communications, \\ University of Oulu, \\ Finland \\ E-mail: rahmat.siregar@oulu.fi,nandana.rajatheva@oulu.fi,matti.latva-aho@ oulu.fi
}

\begin{abstract}
In this study, a quadrature spatial modulation (QSM) based non-orthogonal multiple access (NOMA) for multiuser, named as NOMA-QSM, is proposed. The main idea of NOMA-QSM is to superimpose several constellation symbols to be transmitted simultaneously via some selected antenna indexes by distinguishing power domain. Different signal domain such as antenna indexes and constellation symbols is then perceived at each receiver. Consequently, both connectivity and spectral efficiency can be improved by NOMA-QSM. As performance measures, spectral efficiency, connectivity and bit error rate (BER) are evaluated by simulations. The result shows that the proposed NOMA-QSM scheme outperforms the existing schemes.
\end{abstract}

Index Terms-quadrature spatial modulation (QSM), nonorthogonal multiple access (NOMA), bit error rate (BER), spectral efficiency.

\section{INTRODUCTION}

$\mathbf{T}$ HE future wireless communication system is challenged to provide a massive connectivity since the number of user connected to the network is increased dramatically. Moreover, the availability of wireless resource is narrowed by the time as the need for high system capacity is also increase. To overcome this problem, the future wireless communication such as $5 \mathrm{G}$ is required to provide advantages, such as robust error performance, massive connectivity and high spectral efficiency [1].

In non-orthogonal multiple access (NOMA), multiple users are allowed to simultaneously utilize the resources such as time and frequency. The signals of these users are superimposed with different power allocation at the transmitter [2]-[8].

Quadrature spatial modulation (QSM) is recently proposed to overcome the weaknesses of the former antenna index modulation technique such as spatial modulation (SM) and space shift keying (SSK). In QSM, real and imaginary part of constellation symbol are utilized to transmit through antenna indexes depending on the incoming bit stream [9]-[13]. Since the signals are always orthogonal, inter-carrier interference (ICI) is entirely avoided.

Recently, many studies have been proposed for multi-user antenna index modulation combined with NOMA. In [14], a combination between SM and NOMA technique for uplink transmission for multi-user scenario was proposed. However, the system can only cover maximum two users using the same resources. NOMA-SM for vehicle-to-vehicle communication was also investigated in [15]. In the study, two phases are considered to exploit NOMA and SM technique. The idea is to perform NOMA-SM at the phase two so that more data can be conveyed. However, the system also can only cover maximum two users at the same resources. Moreover, NOMA combined with generalized space shift keying (GSSK) was proposed in [16]. Several users can be covered in this system. Signals from several NOMA users are superimposed and then transmitted through the antenna index that selected from GSSK users. Spectral efficiency and complexity analysis were presented in this study. However, the number of bit per carrier unit (bpcu) which can be conveyed is lesser compared to the proposed system with the same system configuration. Lately, bit allocation approach of SM for multi-user scenario was also proposed in [17]. This system allocates block of information bits to several users to establish multi-user connection. However, number of connected users are limited by information bits can be provided by BS.

In this study, QSM combined with NOMA for multi-user communication is proposed. In the proposed system, the real and imaginary part of constellation symbol of NOMA users are modulated into antenna indexes defined from QSM users. The signals are then superimposed by distinguishing the power between the NOMA users. Consequently, each user receives the same superimposed symbols. At the receiver, each user will perceive its-own signal accordingly. As results, the bit error rate (BER) performance is analyzed on each user and overall system. As error floor exists when the fixed power allocation is applied, a dynamic power allocation is utilized. As the result, the error floor is successfully mitigated. The spectral efficiency analysis is also presented to analyze the proposed system performance. The result shows that the proposed system outperforms the whole existing technique such as NOMA-GSSK in [16].

\section{SySTEM MODEL OF NOMA AND QSM}

\section{A. System Model of Downlink NOMA}

In downlink NOMA, multiple users are allowed to simultaneously utilize several domains (e.g. time, frequency and code) while the signal of these users is superimposed by distinguishing power domain [2]-[8]. The principal of downlink NOMA is shown in Fig. 1. $\mathrm{N}$ users are paired and superimposed to exploit the same domain simultaneously by 


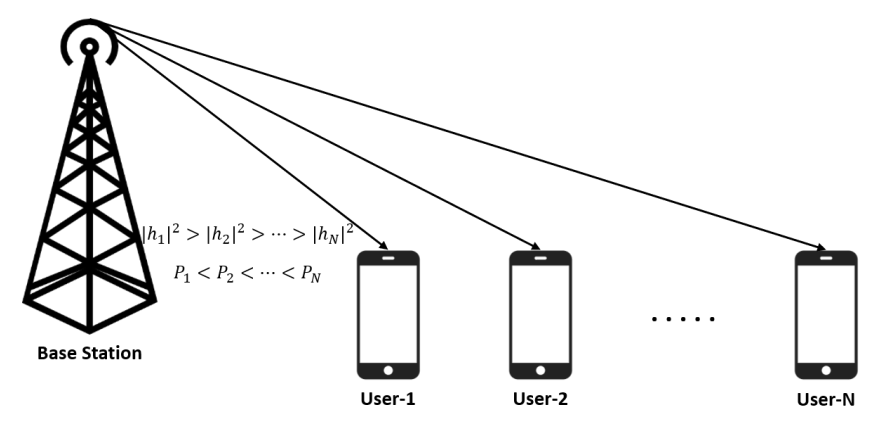

Fig. 1: Downlink NOMA system

allocating different power. At transmitter, the base station (BS) transmits a superimposed signal as given as

$$
x_{\text {noma }}=\sum_{i=1}^{N} p_{i} s_{i}
$$

where $p_{i}$ and $s_{i}$ are allocated power and transmitted symbol of the $i$-th user, respectively, and $E\left[\left|s_{i}\right|^{2}\right]=1$. It is also defined that BS total transmit power is $P$ where $\sum_{i=1}^{N} p_{i}=P$. At each user, the received signal is expressed as

$$
y_{i}=h_{i} x_{n o m a}+w_{i}
$$

where $h_{i}$ and $w_{i}$ are the complex channel response and additive white gaussian noise (AWGN) of $i$-th user, respectively. It is assumed that the channel gain of user- 1 is greater than channel gain of user-2 and so on, $\left|h_{1}\right|^{2}>\left|h_{2}\right|^{2}>\ldots>\left|h_{N}\right|^{2}$. It is also assumed that the allocated power of user 1 is smaller than the allocated power of user 2 , and so on, $p_{1}<p_{2}<\ldots<p_{N}$. For detection scenario, successive interference cancellation (SIC) is performed at each user. At $i$-th user, SIC is utilized to eliminate users with higher allocated power and consider users with lower allocated power as noise. Thus, SIC is performed $N-i$ times at user- $i$ by eliminating the strongest user first, then the second and so on. Therefore, signal at $i$-th user after SIC process can be expressed as

$$
\hat{y}_{i}=y_{i}-h_{i}\left(\sum_{k=i+1}^{N} p_{k} s_{k}\right)
$$

Finally, maximum likelihood estimation (MLE) is used to define user's transmitted symbol. The estimated symbol of user- $i$ is defined as

$$
\hat{s}_{i}=\underset{m}{\arg \min }\left\|\hat{y}_{i}-h_{i} s_{m}\right\|^{2}
$$

where $m=1, \ldots, M$ and $M$ is symbol size (e.g. constellation size).

\section{B. System Model of QSM}

QSM utilizes the real and imaginary part of the constellation symbol to be transmitted through antenna index which is selected independently defined by the incoming bit stream [9][13]. Thus, total number of bpcu can be conveyed at each transmission is

$$
n_{q s m}=\log _{2}\left(N_{t}^{2} M\right) .
$$

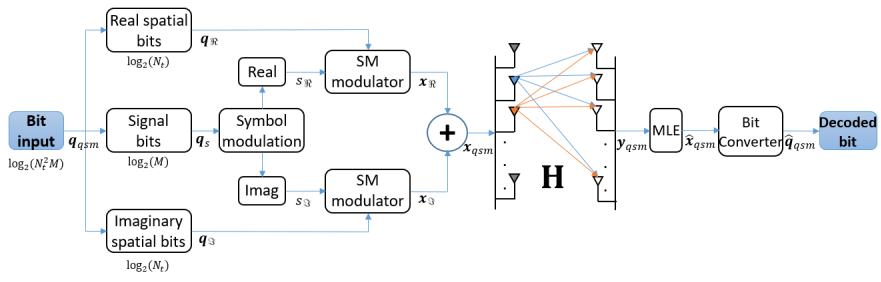

Fig. 2: QSM system

where $N_{t}$ is number of transmit antenna.

As shown in Figure 2, incoming bit stream in vector $\boldsymbol{q}_{q s m}$ of length $n_{q s m}$ is distributed into three vector blocks; $\boldsymbol{q}_{\mathfrak{R}}$ is a vector containing bits to select an antenna for transmitting the real part of the constellation symbol, $\boldsymbol{q}_{s}$ is a vector containing bits to define the constellation symbol and $\boldsymbol{q}_{\mathfrak{J}}$ is a vector containing bits to select an antenna for transmitting the imaginary part of the constellation symbol. In symbol modulation, complex constellation symbol $s=s_{\mathfrak{R}}+j s_{\mathfrak{J}}$ is produced. Both real part $s_{\mathfrak{R}}$ and imaginary part $s_{\mathfrak{J}}$ of the constellation symbol are independently chosen from the selected antenna defined by bit combination in vector $\boldsymbol{q}_{\mathfrak{R}}$ and vector $\boldsymbol{q}_{\mathfrak{J}}$, respectively. Further, in vector $\boldsymbol{x}_{\mathfrak{R}}=\left[\begin{array}{llll}x_{\mathfrak{R} 1} & x_{\mathfrak{R} 2} & \ldots & x_{\mathfrak{R} N_{t}}\end{array}\right]^{T}$ and $\boldsymbol{x}_{\mathfrak{I}}=\left[\begin{array}{llll}x_{\mathfrak{I} 1} & x_{\mathfrak{I} 2} & \ldots & x_{\mathfrak{I} N_{t}}\end{array}\right]^{T}$, there is one element containing $s_{\mathfrak{R}}$ and $s_{\mathfrak{J}}$ in each vector which indicates the transmit antenna index for real and imaginary part, respectively. The $a_{\mathfrak{R}}$-th element in vector $\boldsymbol{x}_{\mathfrak{R}}$ is $x_{\mathfrak{R} a_{\mathfrak{R}}}$ where $a_{\mathfrak{R}}$ is the mapped transmit antenna index $a_{\mathfrak{R}} \in\left[1: N_{t}\right]$. On the other hand, the $a_{\mathfrak{J}}$-th element in vector $\boldsymbol{x}_{\mathfrak{Y}}$ is $s_{\mathfrak{J} a_{\mathfrak{I}}}$ where $a_{\mathfrak{J}}$ is the mapped transmit antenna index $a_{\mathfrak{J}} \in\left[1: N_{t}\right]$. Afterwards, vector $\boldsymbol{x}_{\mathfrak{R}}$ and vector $\boldsymbol{x}_{\mathfrak{I}}$ are summed up resulting new vector $\boldsymbol{x}_{q s m}$. Consequently, the number of activated antenna is either one or two depended on the incoming bit stream in antenna selection part. Vector $\boldsymbol{x}_{q s m}$ is then transmitted over the channel matrix $\mathbf{H}$ of length $N_{r} \times N_{t}$ as written as follows

$$
\mathbf{H}=\left[\begin{array}{cccc}
h_{1,1} & . . & . . & h_{1, N_{t}} \\
: & . & & : \\
: & & & . \\
h_{N_{r}, 1} & . . & . . & h_{N_{r}, N_{t}}
\end{array}\right]
$$

where $N_{r}$ is number of receive antenna, and $h_{u, v}$ is complex channel response, $u \in\left[1: N_{t}\right]$ and $v \in\left[1: N_{t}\right]$. Thus, the received signal at receiver can be expressed as

$$
\boldsymbol{y}_{q s m}=\mathbf{H} \boldsymbol{x}_{q s m}+\boldsymbol{w}=\left(\mathbf{H} \boldsymbol{x}_{\mathfrak{R}}+\mathbf{H} \boldsymbol{x}_{\mathfrak{Y}}\right)+\boldsymbol{w}
$$

where $\boldsymbol{w}$ is AWGN noise vector.

By assuming perfect channel knowledge at receiver, the received signal is estimated using the optimum MLE as shown as

$$
\begin{aligned}
\hat{\boldsymbol{x}}_{q s m} & =\left[\hat{a}_{\mathfrak{R}}, \hat{a}_{\mathfrak{J}}, \hat{s}_{\mathfrak{R}}, \hat{s}_{\mathfrak{J}}\right] \\
& =\underset{a_{\mathfrak{R}}, a_{\mathfrak{J}}, s_{\mathfrak{R}}, s_{\mathfrak{I}}}{\arg \min }\left\|\boldsymbol{y}_{Q S M}-\left(\mathbf{H} \boldsymbol{x}_{a_{\mathfrak{R}}, s_{\mathfrak{R}}}+\mathbf{H} \boldsymbol{x}_{a_{\mathfrak{I}}, s_{\mathfrak{J}}}\right)\right\|^{2}
\end{aligned}
$$

where $s_{\mathfrak{R}}, s_{\mathfrak{I}}=1, \ldots, M$ and $\boldsymbol{x}_{a_{\mathfrak{R}}, s_{\mathfrak{R}}}$ represents real part of symbol number $s_{\mathfrak{R}}$-th transmitted through antenna number $a_{\mathfrak{R}}$ while the other elements remain zero and $\boldsymbol{x}_{a_{\mathfrak{J}}, s_{\mathfrak{J}}}$ represents imaginary part of symbol number $s_{\mathfrak{J}}$-th transmitted through 


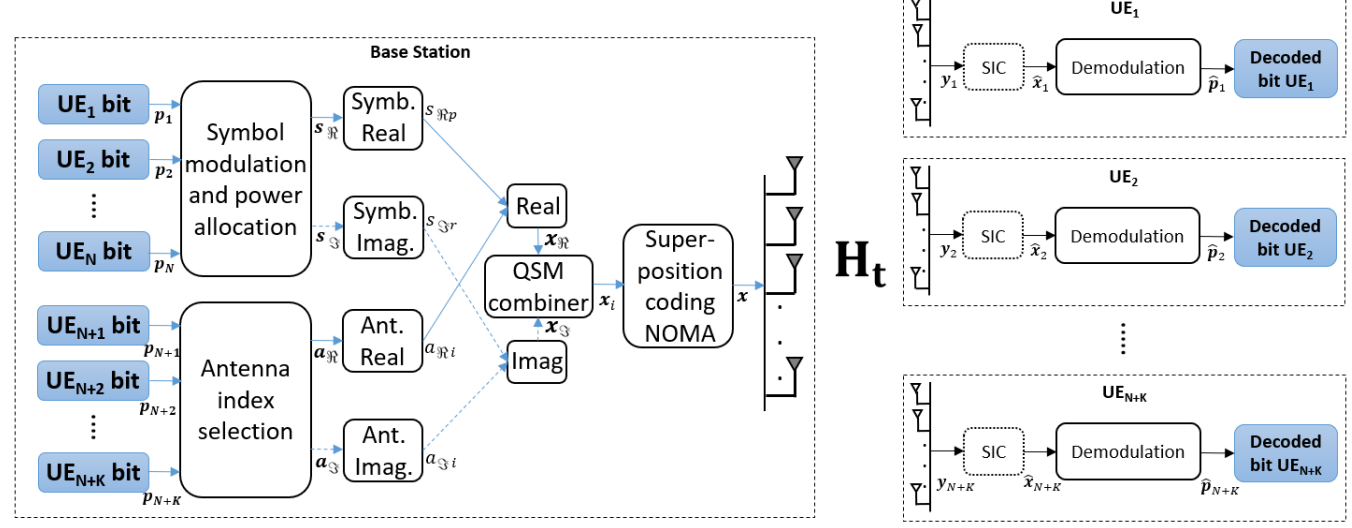

Fig. 3: Proposed System Model

antenna number $a_{\mathfrak{I}}$ while the other elements remain zero. $\hat{a}_{\mathfrak{R}}, \hat{a}_{\mathfrak{I}}, \hat{s}_{\mathfrak{R}}, \hat{s}_{\mathfrak{Y}}$ are the detected antenna index of real part, the detected antenna index of imaginary part, the detected real part symbol and the detected imaginary part symbol, respectively. Finally, vector $\hat{\boldsymbol{x}}_{q s m}$ is converted into bits.

\section{Multi-User QSM BASEd NOMA}

In this chapter, we explain our proposed system using QSM based NOMA for multi-user communication. Assume $N+K$ users to be uniformly distributed in a cell where the constellation symbols of the $\mathrm{N}$ users are transmitted through antenna indexes selected from $\mathrm{K}$ users information bit. Further, the $\mathrm{N}$ users are called as NOMA user while $\mathrm{K}$ users are called as QSM user. As an example, when there is one NOMA user in the cell, it is possible to cover two QSM users. On the other hand, it states that $K=2 N$. Therefore, number of bpcu can be conveyed in this cell is defined as

$$
m=N \log _{2}(M)+K \log _{2}\left(N_{t}\right)=\log _{2}\left(M^{N} N_{t}^{K}\right) .
$$

The proposed system is illustrated in Fig. 3. Assume that vector $\boldsymbol{p}_{t}$ is vector containing bits of user $t$. As $N$ users are defined as NOMA user, $\boldsymbol{p}_{1}, \boldsymbol{p}_{2}, \ldots, \boldsymbol{p}_{N}$ contain bits of length $m_{s}=\log _{2}(M)$. While vector $\boldsymbol{p}_{N+1}, \boldsymbol{p}_{N+2}, \ldots, \boldsymbol{p}_{N+K}$ contain bits of length $m_{a}=\log _{2}\left(N_{t}\right)$. $N$ vectors of NOMA users are then modulated to constellation symbol, multiplied with its power and separated to real and imaginary part resulting new vectors $\boldsymbol{s}_{\mathfrak{R}}$ and $\boldsymbol{s}_{\mathfrak{Y}}$ of length $N, \boldsymbol{s}_{\mathfrak{R}}=\left[\begin{array}{llll}p_{1} s_{\mathfrak{R} 1} & p_{2} s_{\mathfrak{R} 2} & \ldots & p_{N} s_{\mathfrak{R} N}\end{array}\right]$ and $\boldsymbol{s}_{\mathfrak{I}}=\left[\begin{array}{lllll}p_{1} s_{\mathfrak{I} 1} & p_{2} s_{\mathfrak{I} 2} & \ldots & p_{N} s_{\mathfrak{I} N}\end{array}\right]$, where $p_{i}$ is allocated power of NOMA user $i$.

Vector $\boldsymbol{a}_{\mathfrak{R}}=\left[a_{\mathfrak{R} 1} a_{\mathfrak{R} 2} \ldots a_{\mathfrak{R} \frac{K}{2}}\right]$ is vector containing antenna indexes to locate each element of vector $\boldsymbol{s}_{\mathfrak{R}}$ as well as vector $\boldsymbol{a}_{\mathfrak{I}}=\left[a_{\mathfrak{J} 1} a_{\mathfrak{I} 2} \ldots a_{\mathfrak{I} \frac{K}{2}}\right]$ is vector containing antenna indexes to locate each element of vector $\boldsymbol{s}_{\mathfrak{Y}}$. Thus, the $p$-th element in vector $\boldsymbol{a}_{\mathfrak{R}}$ is the position of element $p$ of vector $\boldsymbol{s}_{\mathfrak{R}}$ in new vector $\boldsymbol{x}_{\mathfrak{R} i}$. It means that there is only one element in vector $\boldsymbol{x}_{\mathfrak{R} i}$ different from zero. The $r$-th element in vector $\boldsymbol{a}_{\mathfrak{I}}$ is the position of element $r$ of vector $\boldsymbol{s}_{\mathfrak{I}}$ in new vector $\boldsymbol{x}_{\mathfrak{I} i}$. It means that there is only one element in vector $\boldsymbol{x}_{\mathfrak{I} i}$ different from zero. Vector $\boldsymbol{x}_{\mathfrak{R} i}$ and $\boldsymbol{x}_{\mathfrak{S} i}$ are then summed up resulting new vector $\boldsymbol{x}_{i}$. These processes are repeated for $N$ NOMA users and $K$ QSM users. Total $N$ of vector $\boldsymbol{x}_{i}$ are then superimposed resulting vector $\boldsymbol{x}$ to be further transmitted, $\boldsymbol{x}=\boldsymbol{x}_{1}+\boldsymbol{x}_{2}+\ldots+$ $\boldsymbol{x}_{N}$. The transmit antenna activation is defined according to the elements in vector $\boldsymbol{x}$. Vector $\boldsymbol{x}$ is then broadcast over the channel matrix $\mathbf{H}_{t}$, where $\mathbf{H}_{t}$ is channel matrix $\mathbf{H}$ of user $t$. Therefore, the received vector at every receiver is given by

$$
\boldsymbol{y}_{t}=\mathbf{H}_{t} \boldsymbol{x}+\boldsymbol{w}_{t}
$$

where $\boldsymbol{w}_{t}$ is AWGN noise vector of user $t$.

At receiver, successive interference cancellation (SIC) is performed to eliminate user's signal with higher power allocation. As example, three grouped users is illustrated in Fig. 4. Assume that 3 NOMA users are exist in the cell. Therefore, 3 power allocations are needed to be allocated to these users. It is also assumed that group 1's channel gain is lower than group 2 and group $3,\left|h_{\text {group } 1}\right|^{2}<\left|h_{\text {group } 2}\right|^{2}<\left|h_{\text {group } 3}\right|^{2}$. The antenna selection is then defined by other 6 QSM users where the antenna position for real and imaginary symbol of first NOMA user is defined by the first and second of the QSM user, respectively, the antenna position of second NOMA user is defined by the third and fourth of the QSM user, respectively, and so on. Further, the first NOMA user, the first and the second QSM user are grouped in group 1. With the same manner, there will be 3 groups in this example. SIC is not performed by users in group 1 since group 1 has the highest power allocation and considers group 2 and group 3's signal as noise. On the other hand, SIC is performed by group 2 to eliminate group 1's signal. And group 3 also performs SIC to eliminate group 1 and group 2's signal. Further, $\hat{\boldsymbol{x}}_{t}$ is a vector that contains the received signal of $t$-th user after SIC process. Finally, by assuming perfect channel knowledge at the receiver, vector $\hat{\boldsymbol{x}}_{t}$ is estimated using the optimum MLE and resulting new vector $\hat{\boldsymbol{p}}_{t}$. Vector $\hat{\boldsymbol{p}}_{t}$ is a vector containing whole estimated symbol at user $t, \hat{\boldsymbol{p}}_{t}=\left[\begin{array}{llll}\hat{s}_{\mathfrak{R} t} & \hat{s}_{\mathfrak{I} t} & \hat{a}_{\mathfrak{R} t} & \hat{a}_{\mathfrak{I} t}\end{array}\right]$. MLE process is expressed as

$$
\begin{aligned}
\hat{\boldsymbol{p}}_{t} & =\left[\hat{s}_{\mathfrak{R} t}, \hat{s}_{\mathfrak{I} t}, \hat{a}_{\mathfrak{R} t}, \hat{a}_{\mathfrak{I} t}\right] \\
& =\underset{s_{\mathfrak{R}}, s_{\mathfrak{I}}, a_{\mathfrak{K}}, a_{\mathfrak{I}}}{\arg \min }\left\|\hat{\boldsymbol{x}}_{t}-\left(\mathbf{H}_{t} \boldsymbol{x}_{s_{\mathfrak{R}}, a_{\mathfrak{R}}}+\mathbf{H}_{t} \boldsymbol{x}_{s_{\mathfrak{I}}, a_{\mathfrak{I}}}\right)\right\|^{2}
\end{aligned}
$$




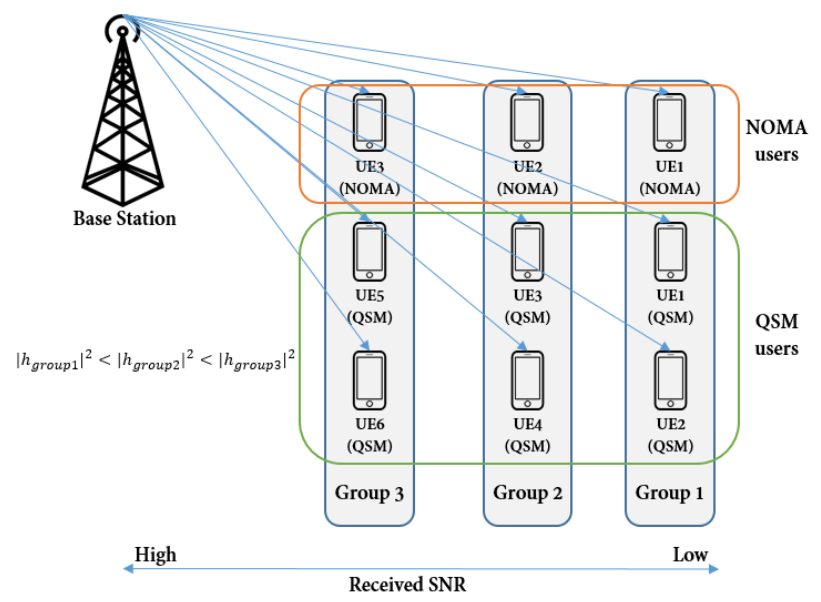

Fig. 4: Three groups example of multi-user QSM NOMA

where $s_{\mathfrak{R}}, s_{\mathfrak{I}}=1, \ldots, M$ are the real and imaginary part of constellation symbol, respectively, and $a_{\mathfrak{R}}, a_{\mathfrak{I}}=1, \ldots, N_{t}$ are the antenna index of real and imaginary symbol, respectively. Vector $\boldsymbol{x}_{s_{\mathfrak{K}}, a_{\mathfrak{K}}}$ represents real part of symbol $s_{\mathfrak{R}}$ that transmitted through antenna number $a_{\mathfrak{R}}$ while the other elements remain zero and $\boldsymbol{x}_{s_{\mathfrak{Y}}, a_{\mathfrak{Y}}}$ represents imaginary part of symbol $s_{\mathfrak{I}}$ that transmitted through antenna number $a_{\mathfrak{I}}$ while the other elements remain zero. And $\hat{s}_{\mathfrak{R} t}, \hat{s}_{\mathfrak{I} t}, \hat{a}_{\mathfrak{R} t}, \hat{a}_{\mathfrak{I} t}$ are the estimated real symbol, the estimated imaginary symbol, the estimated antenna index of real part and the estimated antenna index of imaginary part of user $t$, respectively. Further, the $t$-th user will only consider the specific element in vector $\hat{\boldsymbol{p}}_{t}$ as its estimated symbol. In the previous example, the first NOMA user considers the estimated symbol $\hat{s}_{\mathfrak{K}}$ and $\hat{s}_{\mathfrak{I}}$ as its information. While the first and second QSM user consider the estimated symbol $\hat{a}_{\mathfrak{R}}$ and $\hat{a}_{\mathfrak{J}}$ as their information, respectively.

\section{Simulation Result}

In this section, simulation result of bit error rate (BER) and spectral efficiency for the proposed system are presented. It is assumed that the signal is transmitted over Rayleigh fading channel. Perfect channel knowledge and perfect SIC at each receiver are also considered in this simulation. MLE is used to estimate the received signal at each user. It is also assumed that time and frequency are perfectly synchronized at both transmitter and receiver.

The spectral efficiency analysis is presented is Fig. 5. The result is compared with several existing techniques. In MIMONOMA system, the whole antennas are activated to transmit symbol through each antenna. This leads to high inter-channel interference (ICI) at receiver. While in NOMA-SSK system, since only one antenna is allowed to be activated at a time, there is only one user can act as SSK user which leads to lack of number of covered users compared to the other systems. In NOMA-GSSK system as proposed in [16], every symbol of NOMA user can only provide one GSSK user. While in the proposed system, a constellation symbol of NOMA user can provide other two QSM users with the same amount of

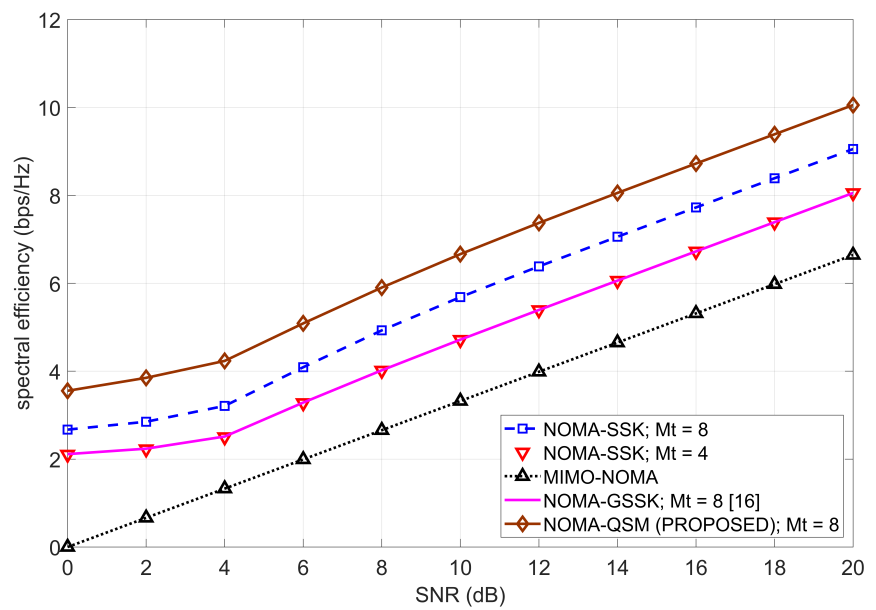

Fig. 5: Spectral efficiency performance of the proposed system compared with the existing system

information compared to NOMA-GSSK. For those reasons, the proposed system outperforms the whole existing technique with the same system configuration over the whole SNR range.

Number of maximum connected users is presented in Fig. 6. Arbitrary $M$ and $N t$ can be utilized without affecting the result. Where $N \alpha$ is number of combined signal using superposition NOMA, it is shown that NOMA-QSM outperforms the existing system and increase exponentially by increasing $N \alpha$. However, increasing $N \alpha$ will affect the number of SIC process which has to perform and the amount of interference appear at the high allocated power user will increase as well.

Fig. (7a) shows the BER performance of six users consists of 2 NOMA users and 4 QSM users. Fixed power allocation is applied in this simulation where $p_{1}=p_{2}=p_{3}=0.9$ and $p_{4}=p_{5}=p_{6}=0.1$. User 1 , user 2 and user 3 are grouped as group 1 where user 1 is assigned as NOMA user and user 2 and user 3 are assigned as QSM user. At each receiver user, user 1 considers constellation symbol $\hat{s}_{\mathfrak{R}}$ and $\hat{s}_{\mathfrak{I}}$ as its information, user 2 considers real transmit antenna $\hat{a}_{\mathfrak{R}}$ as its information and user 3 considers imaginary transmit antenna $\hat{a}_{\mathfrak{I}}$ as its information. SIC is not performed by users in group 1 since highest power allocation is allocated to these users. User 4, user 5 and user 6 are grouped as group 2 where user 4 is assigned as NOMA user and user 5 and user 6 are assigned as QSM user. At each receiver, user 4 considers constellation symbol $\hat{s}_{\mathfrak{R}}$ and $\hat{s}_{\mathfrak{J}}$ as its information, user 5 considers real transmit antenna $\hat{a}_{\mathfrak{R}}$ as its information and user 6 considers imaginary transmit antenna $\hat{a}_{\mathfrak{Y}}$ as its information. SIC is performed by user in group 2 to eliminate NOMA signal of group 1. It is also assumed that channel gain in the same group is approximately same and channel gain of users in group 1 is lower than channel gain of users in group 2, $\left|h_{\text {user } 1}\right|^{2} \approx\left|h_{\text {user } 2}\right|^{2} \approx\left|h_{\text {user } 3}\right|^{2}<\left|h_{\text {user } 4}\right|^{2} \approx\left|h_{\text {user } 5}\right|^{2} \approx$ $\left|h_{\text {user } 6}\right|^{2}$.

In Fig. (7a), it is shown that the BER performance of users in group 1 outperforms users in group 2 at relatively low signal to noise ratio (SNR) since the power allocation of users 


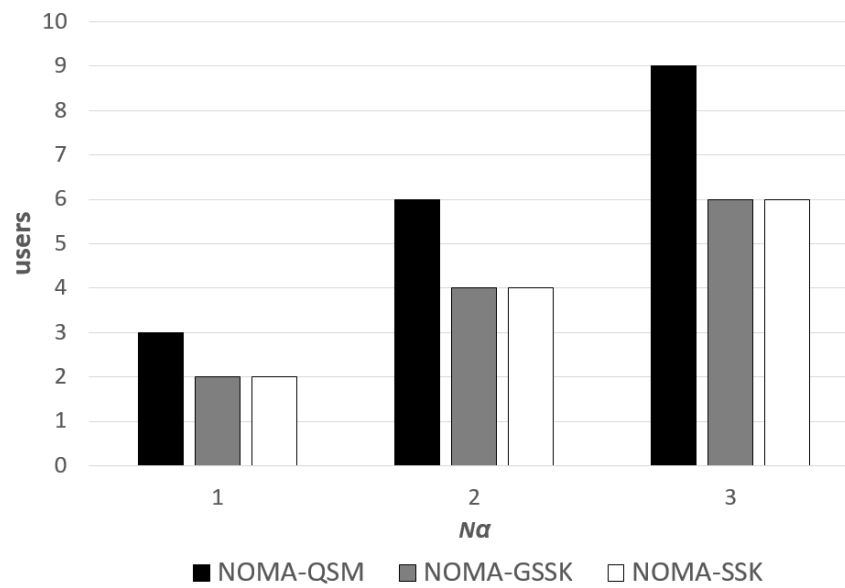

Fig. 6: Number of maximum connected user comparison between the proposed and the existing system

in group 1 is higher than users in group 2. While at high SNR region, users in group one suffer from error floor which cause users in group 2 outperforms users in group 1. This due to the interference resulting from users in group 2 can not be perfectly canceled. Thus, at the relatively low SNR, where the AWGN noise power is higher than the interference power from users in group 2, the AWGN noise can still be reduced by increasing the received SNR. When the AWGN noise power becomes lower than the interference power at high SNR, the interference still present with the same power level. Thus, interference that presents at users in group 1 remain the same and can not be reduced by only increasing the received SNR. In other words, fixed power allocation scenario causes a stagnant residual interference from lower allocated power users to higher allocated power users. Consequently, error floor presents at high SNR for all users in group 1. On the other hand, users in group 2 does not have any additional interference from users in group 1 since perfect SIC is considered. The solid line without marker in Fig. (7a) shows the BER performance of overall system. The error floor at the line presents because of the users in group 1 .

In Fig. (7b), dynamic power allocation is performed to mitigate error floor at users in group 1 . The power allocation for NOMA user in group 2 follows [18, Eq. (17)] as shown as

$$
p_{2}=\frac{\beta_{\text {user } 1}}{W+1}+\frac{\beta_{\text {user } 2}}{V+1}
$$

where $W=\sqrt{1+\rho\left|h_{\text {user } 1}\right|^{2}}, V=\sqrt{1+\rho\left|h_{\text {user } 2}\right|^{2}}$ where $\rho$ is transmit SNR and $\beta_{\text {user } 1}, \beta_{\text {user } 2}$ are two constant coefficients where $\beta_{\text {user } 1}+\beta_{\text {user } 2}=1$ and $0 \leq \beta_{\text {user }-i} \leq 1$ for $i=1,2$. While the power allocation for NOMA user in group 1 follows

$$
p_{1}=1-p_{2} .
$$

In Fig. (7b), it is shown that users in group 2 outperform users in group 1 at whole SNR range due to the channel gain of users in group 2 is higher than channel gain of users in group $1,\left|h_{\text {group } 2}\right|>\left|h_{\text {group } 1}\right|$. By performing dynamic power

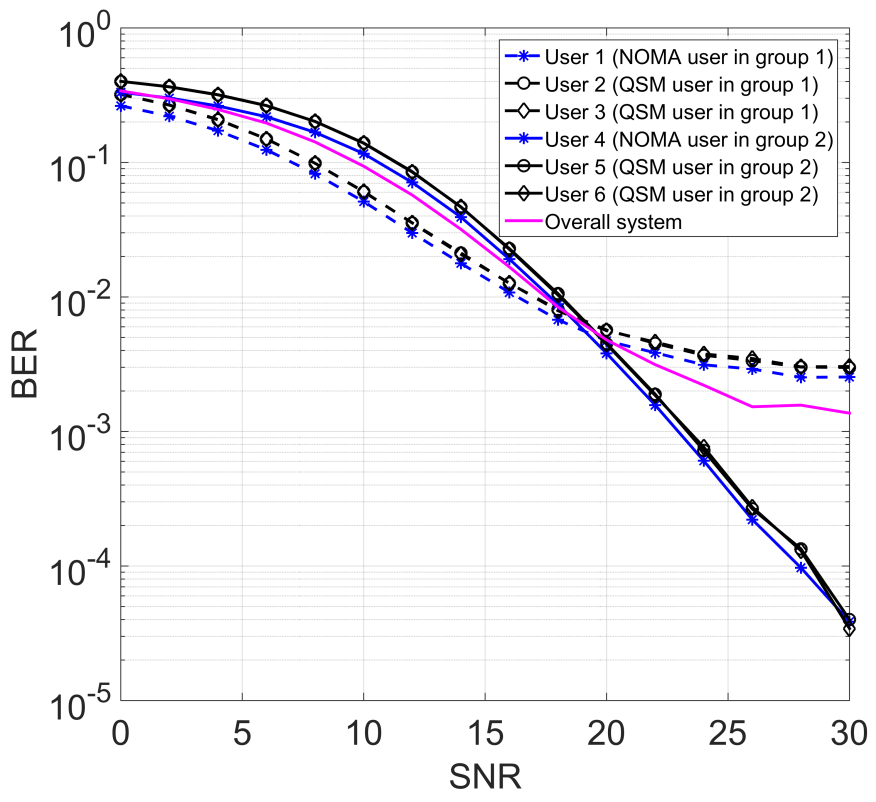

(a) Fixed power allocation

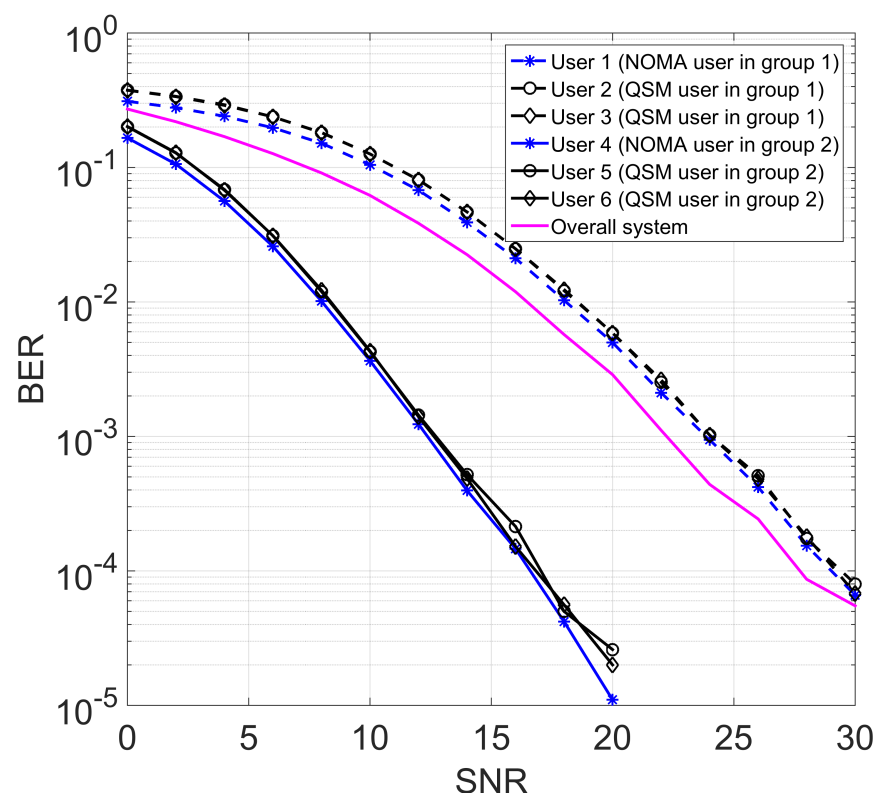

(b) Dynamic power allocation

Fig. 7: BER performance of the proposed system for 6 users scenario (2 NOMA users and 4 QSM users) using both fixed and dynamic power allocation where $M=4$ and $N t=4$

allocation, the error floor of users in group 1 observed in Fig. (7a) is successfully mitigated. Consequently, error floor in the overall system is also mitigated.

\section{CONClusion}

In this study, we propose NOMA-QSM for multi-user scenario. In the proposed system, a superimposed signal of several constellation symbols is transmitted simultaneously by distinguishing the power domain. Different signal domain is then perceived at each receiver. A robust BER performance 
is offered in this system when dynamic power allocation is utilized. On the other hand, the spectral efficiency analysis shows that the proposed system outperforms the other existing techniques such as MIMO-NOMA system, NOMA-SSK system and NOMA-GSSK system [16] by using the same system configuration over the whole SNR range. In addition, it was shown that the proposed system can cover more users compared with the existing systems.

Future work will consider coded schemes using the proposed system. We will analyze performance measurements under several channel conditions and more practical scenario.

\section{ACKNOWLEDGMENT}

This work has been financially supported in part by the 6Genesis (6G) Flagship project (grant 318927).

\section{REFERENCES}

[1] D. Jiang and G. Liu, "An overview of $5 \mathrm{G}$ requirements," in $5 G$ Mobile Communications. Springer, 2017, pp. 3-26.

[2] L. Dai, B. Wang, Z. Ding, Z. Wang, S. Chen, and L. Hanzo, "A survey of non-orthogonal multiple access for 5g," IEEE Communications Surveys Tutorials, pp. 1-1, 2018.

[3] Y. Alsaba, C. Y. Leow, and S. K. A. Rahim, "Full-duplex cooperative non-orthogonal multiple access with beamforming and energy harvesting," IEEE Access, vol. 6, pp. 19726-19738, 2018.

[4] W. Wang, Y. Liu, Z. Luo, T. Jiang, Q. Zhang, and A. Nallanathan, "Toward cross-layer design for non-orthogonal multiple access: A qualityof-experience perspective," IEEE Wireless Communications, vol. 25, no. 2, pp. 118-124, April 2018.

[5] D. Wan, M. Wen, F. Ji, H. Yu, and F. Chen, "Non-orthogonal multiple access for cooperative communications: Challenges, opportunities, and trends," IEEE Wireless Communications, vol. 25, no. 2, pp. 109-117, April 2018.

[6] L. Yuan, J. Pan, N. Yang, Z. Ding, and J. Yuan, "Successive interference cancellation for ldpc coded non-orthogonal multiple access systems," IEEE Transactions on Vehicular Technology, pp. 1-1, 2018.

[7] G. Lv, X. Li, R. Shang, P. Xue, and Y. Jin, "Dynamic resource allocation for uplink non-orthogonal multiple access systems," IET Communications, vol. 12, no. 6, pp. 649-655, 2018.

[8] F. W. Murti, R. F. Siregar, and S. Y. Shin, "Su-mimo based uplink nonorthogonal multiple access for $5 \mathrm{~g}$," Journal of Network and Computer Applications, vol. 110, pp. 87 - 96, 2018.

[9] R. Mesleh, S. S. Ikki, and H. M. Aggoune, "Quadrature spatial modulation," IEEE Transactions on Vehicular Technology, vol. 64, no. 6, pp. 2738-2742, June 2015.

[10] R. Mesleh, S. Althunibat, and A. Younis, "Differential quadrature spatial modulation," IEEE Transactions on Communications, vol. 65, no. 9, pp. 3810-3817, Sept 2017.

[11] L. Wang, Z. Chen, Z. Gong, and M. Wu, "Diversity-achieving quadrature spatial modulation," IEEE Transactions on Vehicular Technology, vol. 66, no. 12, pp. 10764-10775, Dec 2017.

[12] B. Vo and H. H. Nguyen, "Improved quadrature spatial modulation," in 2017 IEEE 86th Vehicular Technology Conference (VTC-Fall), Sept 2017 , pp. $1-5$.

[13] N. Pillay and H. Xu, "Quadrature spatial media-based modulation with rf mirrors," IET Communications, vol. 11, no. 16, pp. 2440-2448, 2017.

[14] R. F. Siregar, F. W. Murti, and S. Y. Shin, "Combination of spatial modulation and non-orthogonal multiple access using hybrid detection scheme," in 2017 Ninth International Conference on Ubiquitous and Future Networks (ICUFN), July 2017, pp. 476-481.

[15] Y. Chen, L. Wang, Y. Ai, B. Jiao, and L. Hanzo, "Performance analysis of noma-sm in vehicle-to-vehicle massive mimo channels," IEEE Journal on Selected Areas in Communications, vol. 35, no. 12, pp. 2653-2666, Dec 2017.

[16] J. W. Kim, S. Y. Shin, and V. C. M. Leung, "Performance enhancement of downlink noma by combination with gssk," IEEE Wireless Communications Letters, pp. 1-1, 2018.
[17] R. F. Siregar, F. W. Murti, and S. Y. Shin, "Bit allocation approach of spatial modulation for multi-user scenario," Journal of Network and Computer Applications, vol. 127, pp. 1 - 8, 2019.

[18] Z. Yang, Z. Ding, P. Fan, and N. Al-Dhahir, "A general power allocation scheme to guarantee quality of service in downlink and uplink noma systems," IEEE Transactions on Wireless Communications, vol. 15, no. 11, pp. 7244-7257, Nov 2016. 